University of Nebraska - Lincoln

DigitalCommons@University of Nebraska - Lincoln

Faculty Publications from the Department of Electrical \& Computer Engineering, Department Electrical and Computer Engineering

9-15-1997

\title{
Phase and microstructure investigations of boron nitride thin films by spectroscopic ellipsometry in the visible and infrared spectral range
}

\author{
Eva Franke \\ Institute of Surface Modification, D-04303 Leipzig, Germany, efranke3@unl.edu \\ Mathias Schubert \\ University of Nebraska-Lincoln, mschubert4@unl.edu \\ Horst Neumann \\ Institute of Surface Modification, D-04303 Leipzig, Germany \\ Thomas E. Tiwald \\ University of Nebraska-Lincoln \\ Daniel W. Thompson \\ University of Nebraska-Lincoln, dthompson2@unl.edu \\ See next page for additional authors \\ Follow this and additional works at: https://digitalcommons.unl.edu/electricalengineeringfacpub \\ Part of the Electrical and Computer Engineering Commons
}

Franke, Eva; Schubert, Mathias; Neumann, Horst; Tiwald, Thomas E.; Thompson, Daniel W.; Woollam, John A.; Hahn, Jens; and Richter, Frank, "Phase and microstructure investigations of boron nitride thin films by spectroscopic ellipsometry in the visible and infrared spectral range" (1997). Faculty Publications from the Department of Electrical and Computer Engineering. 64.

https://digitalcommons.unl.edu/electricalengineeringfacpub/64

This Article is brought to you for free and open access by the Electrical \& Computer Engineering, Department of at DigitalCommons@University of Nebraska - Lincoln. It has been accepted for inclusion in Faculty Publications from the Department of Electrical and Computer Engineering by an authorized administrator of DigitalCommons@University of Nebraska - Lincoln. 


\section{Authors}

Eva Franke, Mathias Schubert, Horst Neumann, Thomas E. Tiwald, Daniel W. Thompson, John A. Woollam, Jens Hahn, and Frank Richter 


\title{
Phase and microstructure investigations of boron nitride thin films by spectroscopic ellipsometry in the visible and infrared spectral range
}

\author{
Eva Franke, ${ }^{\text {a) }}$ Mathias Schubert, and Horst Neumann \\ Institute of Surface Modification, D-04303 Leipzig, Germany
}

Thomas E. Tiwald, Daniel W. Thompson, and John A. Woollam

Center for Microelectronic and Optical Materials Research, and Department of Electrical Engineering, University of Nebraska-Lincoln, Lincoln, Nebraska 68588

\author{
Jens Hahn and Frank Richter \\ Institute of Physics, Technical Univesity Chemnitz-Zwickau, D-09107 Chemnitz, Germany
}

(Received 22 May 1997; accepted for publication 18 June 1997)

\begin{abstract}
Spectroscopic ellipsometry over the spectral range from 700 to $3000 \mathrm{~cm}^{-1}$ and from 1.5 to $3.5 \mathrm{eV}$ is used to simultaneously determine phase and microstructure of polycrystalline hexagonal and cubic boron nitride thin films deposited by magnetron sputtering on (100) silicon. The results are obtained from a single microstructure-dependent model for both infrared and visible-light thin-film anisotropic dielectric functions. The optical behavior of high $c$-BN content thin films is described by an effective medium approximation. We obtain the amount of $h$-BN within high $c$-BN content thin films. A thin oriented nucleation layer between the silicon substrate and the high $c$-BN content layer is demonstrated. The preferential arrangement of the grain $c$ axes within the $h$-BN thin films are found to be dependent on the growth parameters. The results from the infrared and visible spectral range ellipsometry model are compared to each other and found to be highly consistent. (C) 1997 American Institute of Physics. [S0021-8979(97)06618-8]
\end{abstract}

\section{INTRODUCTION}

Various chemical or physical vapor-phase deposition techniques have been successfully used to deposit pure-phase hexagonal $(h)$ or high cubic $(c)$ boron nitride $(\mathrm{BN})$ content thin films. ${ }^{1-7}$ The microstructure of polycrystalline $\mathrm{BN}$ thin films usually consists of mixtures of $c-\left(s p^{3}\right.$-bonded, zincblende structure) and $h$ - $\left(s p^{2}\right.$-bonded, graphite-like structure) $\mathrm{BN}$ as has been reported from high-resolution transmission electron microscopy (HRTEM) in combination with selected area electron diffraction (SAD). ${ }^{8,9}$ The $c$-axis orientations of the $h$-BN grains (grain size: $20-50 \mathrm{~nm}$ ) within pure-phase thin films depend on the growth parameters. ${ }^{10}$ The high $c$-BN content films consist of a characteristic layer structure: A noncubic nucleation layer is followed by a nanocrystalline high $c$-BN content layer (grain size: 10-30 $\mathrm{nm}) .{ }^{8}$ The grain boundaries between the $c$-BN grains have been demonstrated to consist of $h$-BN material. ${ }^{11}$ Because of the well-separated lattice resonance frequencies in $h$-BN and $c$-BN, infrared spectroscopy (IRS) is commonly used to distinguish the phases contained within the BN thin films. Raman spectroscopy and IRS were employed to explore the lattice resonance properties of amorphous, turbostratic, $h$ and $c$-BN. ${ }^{12-19}$ However, the infrared reflectance is a function of the thin-film microstructure as well, and there have been no reports on a quantitative analysis of the thinfilm dielectric function (DF) properties. For a better understanding of BN thin-film reflectivity data, the influence of microstructure on the thin-film DF must be taken into consideration.

First, investigations of the $h$-BN and $c$-BN visible refractive indices were performed on single crystals: hexagonal

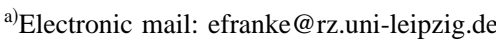

$\mathrm{BN}$ is uniaxially negative with extraordinary refractive index $n_{e}=1.65(E \| c)$, and ordinary index $n_{o}=2.17(E \perp c) .{ }^{20} \mathrm{Cu}-$ bic $\mathrm{BN}$ is optically isotropic $(n=2.13) .{ }^{20} \mathrm{~A}$ few reports exist on the visible-light $\mathrm{BN}$ thin-film optical properties obtained by ellipsometry or transmittance measurements. ${ }^{21-28}$ However, the BN thin-film refractive index was treated as being isotropic. Changes in the refractive index were discussed as being due to $c$-BN content, porosity or thin-film inclusions. In actuality $h$-BN content thin films must be considered as optically anisotropic. Both the infrared thin-film DF and the visible-light effective thin-film refractive indices depend on the thin-film microstructure, i.e., phase composition, layer structure, and average orientations of the $h$-BN grain $c$ axes.

Band structure calculations dealing with dependence on temperature $^{29}$ and hydrostatic pressure ${ }^{30}$ were performed to investigate optical properties of $\mathrm{BN}$ with different crystal structures. Several investigations of bulk and pyrolytic $h$-BN and $c$-BN infrared optical properties have been reported. Geick et al. ${ }^{16}$ observed two characteristic $h$-BN lattice resonance absorptions for electric field polarizations $E$ parallel (out-of-plane: II) and perpendicular (in-plane: $\perp$ ) to the lattice $c$ axis, characterized by their transverse (TO) and longitudinal (LO) optical frequencies $\omega_{\mathrm{TO} \|}=783 \mathrm{~cm}^{-1}, \omega_{\mathrm{LO} \|}=823$ $\mathrm{cm}^{-1}, \omega_{\mathrm{TO} \perp}=1367 \mathrm{~cm}^{-1}$, and $\omega_{\mathrm{LO} \perp}=1610 \mathrm{~cm}^{-1}$, respectively. Gielisse et al. ${ }^{17}$ estimated the characteristic $c$-BN (c) lattice absorptions at resonance frequencies $\omega_{\mathrm{TO} c}=1065$ $\mathrm{cm}^{-1}$ and $\omega_{\mathrm{LO} c}=1340 \mathrm{~cm}^{-1}$, while Eremets et al. ${ }^{18}$ obtained $\omega_{\mathrm{TO} c}=1056 \mathrm{~cm}^{-1}$ and $\omega_{\mathrm{LO} c}=1305 \mathrm{~cm}^{-1}$. Okamoato et al. calculated polarized IRS data from $h$-BN thin films in the case of $c$-axis orientations parallel and perpendicular to the film normal. ${ }^{19}$ However, the Okamoato model does not account for the polycrystalline character of the thin films. 
TABLE I. IRSE and gVASE sample microstructure parameter results.

\begin{tabular}{|c|c|c|c|c|c|c|c|}
\hline \multirow[t]{2}{*}{$\begin{array}{c}\text { Sample/ }\left[U_{B}(\mathrm{~V})\right] \\
\text { Method }\end{array}$} & \multirow[t]{2}{*}{$\begin{array}{c}\text { Top layer } \\
\text { Bottom layer }\end{array}$} & \multicolumn{2}{|c|}{$f_{c}(\%)$} & \multicolumn{2}{|c|}{$\begin{array}{l}d_{1}(\mathrm{~nm}) \\
d_{2}(\mathrm{~nm})\end{array}$} & \multicolumn{2}{|c|}{$\begin{array}{l}\Theta_{1}\left(^{\circ}\right) \\
\Theta_{2}\left(^{\circ}\right)\end{array}$} \\
\hline & & IRSE & gVASE & IRSE & gVASE & IRSE & gVASE \\
\hline$c-\mathrm{BN} 1 /(-150)$ & $\begin{array}{c}c / h-\mathrm{BN} \\
h-\mathrm{BN}\end{array}$ & $83 \pm 5$ & $90 \pm 5$ & $\begin{array}{r}94 \pm 2 \\
3.5 \pm 1\end{array}$ & $106 \pm 2$ & $\begin{array}{l}54.7^{\mathrm{b}} \\
90 \pm 5\end{array}$ & $\begin{array}{c}54.7^{\mathrm{b}} \\
\mathrm{c}\end{array}$ \\
\hline$c-\mathrm{BN} 2 /(-150)$ & $\begin{array}{c}c / h-\mathrm{BN} \\
h-\mathrm{BN}\end{array}$ & $75 \pm 5$ & $88 \pm 5$ & $\begin{array}{r}120 \pm 3 \\
2.5 \pm 1\end{array}$ & $121_{\mathrm{c}} \pm 2$ & $\begin{array}{l}54.7^{\mathrm{b}} \\
90 \pm 5\end{array}$ & $\begin{array}{c}54.7^{\mathrm{b}} \\
\mathrm{c}\end{array}$ \\
\hline$c-\mathrm{BN} 3 /(-150)$ & $\begin{array}{c}c / h-\mathrm{BN} \\
h-\mathrm{BN}\end{array}$ & $70 \pm 5$ & $80 \pm 5$ & $\begin{array}{r}131 \pm 3 \\
24 \pm 1\end{array}$ & $140 \pm 2$ & $\begin{array}{l}54.7^{\mathrm{b}} \\
70 \pm 5\end{array}$ & $\begin{array}{c}54.7^{\mathrm{b}} \\
\mathrm{c}\end{array}$ \\
\hline$h-\mathrm{BN} 1 /(+20)^{\mathrm{a}}$ & $h-\mathrm{BN}$ & & & $278 \pm 4$ & $294 \pm 10$ & $34 \pm 5$ & $40 \pm 5$ \\
\hline$h-\mathrm{BN} 2 /(-60)$ & $h-\mathrm{BN}$ & & & $256 \pm 5$ & $263 \pm 8$ & $54.7^{\mathrm{b}}$ & $57 \pm 5$ \\
\hline$h-\mathrm{BN} 3(-30)$ & $h-\mathrm{BN}$ & & & $520 \pm 8$ & $521 \pm 10$ & $90 \pm 5$ & $90 \pm 5$ \\
\hline
\end{tabular}

${ }^{a}$ Floating potential, no supplied substrate voltage.

' Isotropically averaged $h$-BN.

${ }^{\mathrm{c}}$ No sensitivity provided by gVASE data.

Ellipsometry is a nondestructive optical technique which determines the thin-film complex reflectance ratio $\rho=r_{p} / r_{s}$ $=\tan \Psi \exp [i \Delta]$, where $r_{p}$ and $r_{s}$ are the complex reflectance coefficients of $s$ - and $p$-polarized light, respectively. ${ }^{31} \mathrm{Gen}$ eralized variable angle of incidence spectroscopic ellipsometry (gVASE) allows for anisotropic sample reflectivity and detects measurable " $p$ " and " $s$ " wave conversions. ${ }^{32}$ In order to analyze ellipsometry data one needs to model the sample structure, including the material optical properties.

The present article focuses on the application of infrared spectroscopic ellipsometry (IRSE) and visible-light gVASE in order to determine phase and microstructure of $\mathrm{BN}$ thin films. A single microstructure-dependent, i.e., geometrical, model for the anisotropic visible-light and infrared thin-film DF is applied to analyze different ellipsometry spectra observed on various $\mathrm{BN}$ thin films. We obtain the amount of isotropic averaged $h$-BN within the high $c$-BN content layers, the thickness and preferential $c$-axes orientations of the noncubic nucleation layer, the lattice resonance frequencies, and the average preferential $c$-axis orientations in pure-phase $h$-BN thin films. The results from the infrared and visible spectral range ellipsometry model are compared to each other. The geometrical model of the anisotropic thin-film DF provides highly consistent sample microstructure results, regardless to the spectral range investigated.

\section{EXPERIMENT}

The BN thin films were deposited by a magnetron sputter technique on (100) silicon oriented wafers. Various substrate bias voltages $\left(U_{B}\right)$ were used for the deposition, resulting in polycrystalline pure-phase $h-\mathrm{BN}\left(U_{B}>-100 \mathrm{~V}\right)$ or high $c$-BN content $\left(U_{B}<-100 \mathrm{~V}\right)$ thin films. ${ }^{33}$ Pure $h$-BN and pure boron targets, or pure boron targets only were used as targets during the rf or dc magnetron sputtering process, respectively. The process with boron nitride as target was reactive. Here $10 \mathrm{sccm}$ nitrogen and $90 \mathrm{sccm}$ argon enabled the growth of stoichiometric BN films. The gas flux during the process with the $h$-BN target was $3 \mathrm{sccm}$ nitrogen and $97 \mathrm{sccm}$ argon, and the pressure was $0.2 \mathrm{~Pa}$ in all cases. The substrate temperature was held at $350{ }^{\circ} \mathrm{C}$ during deposition. The samples were investigated by HRTEM and SAD in the [110] and [1-10] pole. ${ }^{10}$ Depending on the growth conditions the $h$-BN $c$ axes show an average preferential orientation changing from nearly parallel $\left(U_{B}=+33 \mathrm{~V}\right)$ to perpendicular $\left(U_{B}=-60 \mathrm{~V}\right)$ to the thin-film normal. The $c-\mathrm{BN}$ thin films consist of the same characteristic layer structure as discussed in Sec. I.

Generalized VASE measurements were carried out on the samples listed in Table I in the photon energy range $1.5 \leqslant \hbar \omega \leqslant 3.5 \mathrm{eV}$ at multiple angles of incidence $\left(\Phi_{a}=55-85^{\circ}\right)$ and multiple azimuth angles $\varphi$ using a rotating-analyzer ellipsometer with automated compensator function (J. A. Woollam Co.), where $\varphi$ is defined as the angle between the plane of incidence and a crystallographic sample orientation parallel to the sample surface. ${ }^{32}$

IRSE measurements were carried out in the wave number range between 700 and $3000 \mathrm{~cm}^{-1}$ at multiple angles of incidence $\left(\Phi_{a}=65-75^{\circ}\right)$. A rotating-polarizer, rotatingcompensator, Fourier-transform based variable angle of incidence spectroscopic ellipsometer was used.

\section{RESULTS AND DISCUSSION}

\section{A. Generalized VASE results}

None of the $h$-BN thin films revealed an observable $p$ - $s$ mode conversion at any sample orientation $\varphi$. The gVASE data explicitly excluded any optical preferential direction parallel to the sample surface. The dependence of the ellipsometric parameters $\Psi$ and $\Delta$ on the incident angle $\Phi_{a}$ was then used to explore any anisotropic properties of the samples. The $c$-BN thin films exhibited isotropic properties consistent with bulk material.

Data for the $h$-BN films were modeled as being uniaxial, and refractive indices were obtained for light polarization parallel $\left(n_{o, \text { eff }}\right)$, and perpendicular ( $\left.n_{e, \text { eff }}\right)$ to the sample surface (Fig. 1). Depending on $U_{B}$, the sign of this anisotropy changes from uniaxially positive $\left(n_{o, \text { eff }}<n_{e, \text { eff }}\right)$ to uniaxially negative $\left(n_{o, \text { eff }}>n_{e, \text { eff }}\right)$. This behavior becomes clear upon considering the $h$-BN microstructure: The microscopic $c$ axes of the $h-\mathrm{BN}$ grains show an average inclination angle $\Theta$ towards the sample normal (Fig. 2). This average inclination is further averaged over the range of in-plane orientations, 
h-BN effective thin-film refractive indices

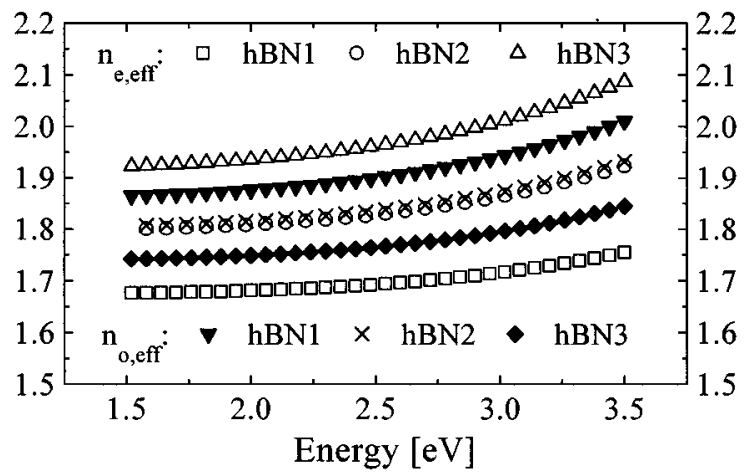

FIG. 1. Effective uniaxial thin-film refractive indices obtained from our $h$-BN samples and analysis of the visible-light gVASE data. Depending on the microstructure the thin-film optical anisotropy appears as uniaxially positive, isotropic, or uniaxially negative.

thus mapping the grain $c$ axes onto the outer shell of a cone. The angle $\Theta$ is then the cone angle spread. This is consistent with SAD patterns which show a preferential orientation of the $h$-BN $c$ axes in both the [110] and the [1-10] pole. ${ }^{10}$ The resulting DF tensor observable by macroscopic techniques is independent of $\varphi$ and is a function of the intrinsic ordinary and extraordinary $h$-BN refractive indices and $\theta::^{10}$

$$
\begin{aligned}
& n_{o, \mathrm{eff}}^{2}=0.25\left[(3+\cos 2 \theta) n_{o}^{2}+(1-\cos 2 \theta) n_{e}^{2}\right], \\
& n_{e, \mathrm{eff}}^{2}=\left(n_{e} \cos \theta\right)^{2}+\left(n_{o} \sin \theta\right)^{2} .
\end{aligned}
$$

Note that $\cos \Theta=1 / \sqrt{3}$ represents randomly averaged $c$-axis orientations, i.e., isotropic $h$-BN. The high $c$-BN content thin-film refractive index is described by the Bruggeman effective medium approximation (EMA): ${ }^{34}$

$$
f_{c} \frac{\varepsilon_{c}-\varepsilon}{\varepsilon_{c}+2 \varepsilon}+f_{b} \frac{\varepsilon_{h}-\varepsilon}{\varepsilon_{h}+2 \varepsilon}=0
$$

where $\varepsilon_{c}=n_{c}^{2}$ and $\varepsilon_{h}=n_{h}^{2}$ are the dielectric functions (refractive indices) of $c$-BN and randomly averaged (isotropic)

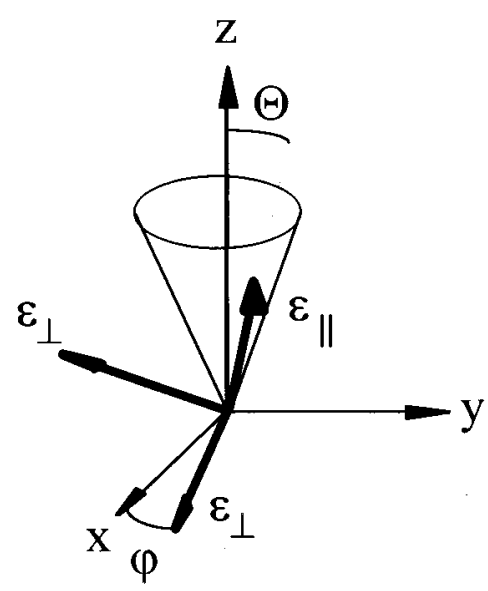

FIG. 2. Model of the geometry of the anisotropic $h$-BN thin-film microstructure from which the dielectric function model used in this work is obtained. The microscopic $h$-BN grain $c$ axes are mapped onto the outer shell of a cone $(0 \leqslant \varphi \leqslant 2 \pi)$. The cone axis is the sample normal. The thin-film optical anisotropy depends on the average $c$-axis grain orientation $\theta$.
$h$-BN (Eqs. (1) at $\Theta=54.7^{\circ}$ ), and $f_{c}$ and $f_{h}=1-f_{c}$ are treated as the respective volume fractions. Two multiplesample regression analysis fits were performed including either the $h$-BN or the $c$-BN samples. The experimental $\Psi$ spectra together with the calculated $\Psi$ data obtained from a multiple-sample fit for the $h$-BN and $c$-BN samples are shown in Fig. 3(a) and Fig. 3(b), respectively. During the regression unique intrinsic refractive indices are coupled together. A $4 \times 4$ matrix formalism which allows for arbitrary sample anisotropy was used to predict the observable data and fit for $n_{o}, n_{e}$, and $n_{c}$, as well as the respective layer thickness and cone angle $\Theta$ ( $h$-BN samples only). ${ }^{35}$ The resulting intrinsic $h$-BN and $c$-BN refractive indices and sample parameters are shown in Fig. 4 and Table I, respectively. Because of the small nucleation layer optical thickness the visible-light gVASE data did not provide enough sensitivity to their structural properties.

\section{B. IRSE results}

The infrared optical properties of $\mathrm{BN}$ are modeled by describing each $h$-BN and $c$-BN lattice resonance absorption as a Lorentz oscillator following Geick et al. ${ }^{16}$ and Eremets et al. ${ }^{18}$ The DF within the reststrahlen spectral range can be written as follows:

$$
\varepsilon_{j}=\varepsilon_{0 j} \omega_{\mathrm{TO} j}^{2} / \omega_{\mathrm{LO} j}^{2}+\varepsilon_{0 j} \omega_{\mathrm{TO} j}^{2} \frac{1-\omega_{\mathrm{TO} j} / \omega_{\mathrm{LO} j}^{2}}{\omega_{\mathrm{TO} j}^{2}-\omega^{2}-i \omega \Gamma},
$$

where $\varepsilon_{0}$ is the static dielectric function, $\omega_{\mathrm{TO}}$ and $\omega_{\mathrm{LO}}$ are the TO and LO optical frequencies and $\Gamma$ is the lattice resonance broadening parameter. The index $j$ refers to the $h$-BN in-plane " $\perp$ " or out-of-plane " $\|$, ," and $c$-BN " $c$ " optical lattice absorptions. The $h$-BN infrared thin-film DF is formally identical to Eqs. (1), where $n_{o \text {,eff }}^{2}=\varepsilon_{o, \text { eff }}$ and $n_{e, \text { eff }}{ }^{2}$ $=\varepsilon_{e \text {,eff }}$ are for DF for polarization parallel and perpendicular to the film normal, and $n_{\|}{ }^{2}=\varepsilon_{\|}$and $n_{\perp}{ }^{2}=\varepsilon_{\perp}$ are the DF for polarization parallel and perpendicular to the bulk $h$-BN $c$ axis, respectively. ${ }^{36}$ As well, the high $c$-BN content thin-film DF can be described by the same EMA model [Eq. (2)] which is used for the thin-film visible-light refractive indices. Here, we assume again randomly oriented $s p^{2}$-bonded material at the $c$-BN grain boundaries (i.e., $\varepsilon_{o, \text { eff }}=\varepsilon_{e, \text { eff }}=\varepsilon_{h}$ at $\Theta=54.7^{\circ}$ ).

The IRSE data acquired from the samples listed in Table I were analyzed using a detailed line shape analysis for modeling the thin-film DF, as presented in Eqs. (1)-(3). Figure 5 (a) shows generated and experimental data ( $\Psi$ only) from our $h$-BN samples. Data from one incident angle are presented only, to avoid confusion between otherwise multiple plots. The generated data were obtained from the best fit using the $h$-BN DF parameters $\left(\varepsilon_{0 \|}, \omega_{\mathrm{TO} \|}, \omega_{\mathrm{LO} \|}, \Gamma_{\|}, \varepsilon_{0 \perp}\right.$, $\left.\omega_{\mathrm{TO} \perp}, \omega_{\mathrm{LO} \perp}, \Gamma_{\perp}\right)$, the cone angle $\Theta$, and layer thickness $d$. The different spectra for the three samples are attributed to different $h$-BN grain $c$-axes orientations. The pronounced peaks in $\Psi$ at $\omega=\omega_{\mathrm{TO} \|}$ and $\omega_{\mathrm{TO} \perp}$ are due to the thin-film TO phonon modes. The minima in $\Psi$ are due to the loss of $p$-polarized reflectance for incident light-wave frequencies above the LO mode frequencies (Berreman effect) ${ }^{37}$ which shifts as a function of $\Theta$. A more detailed discussion of the 

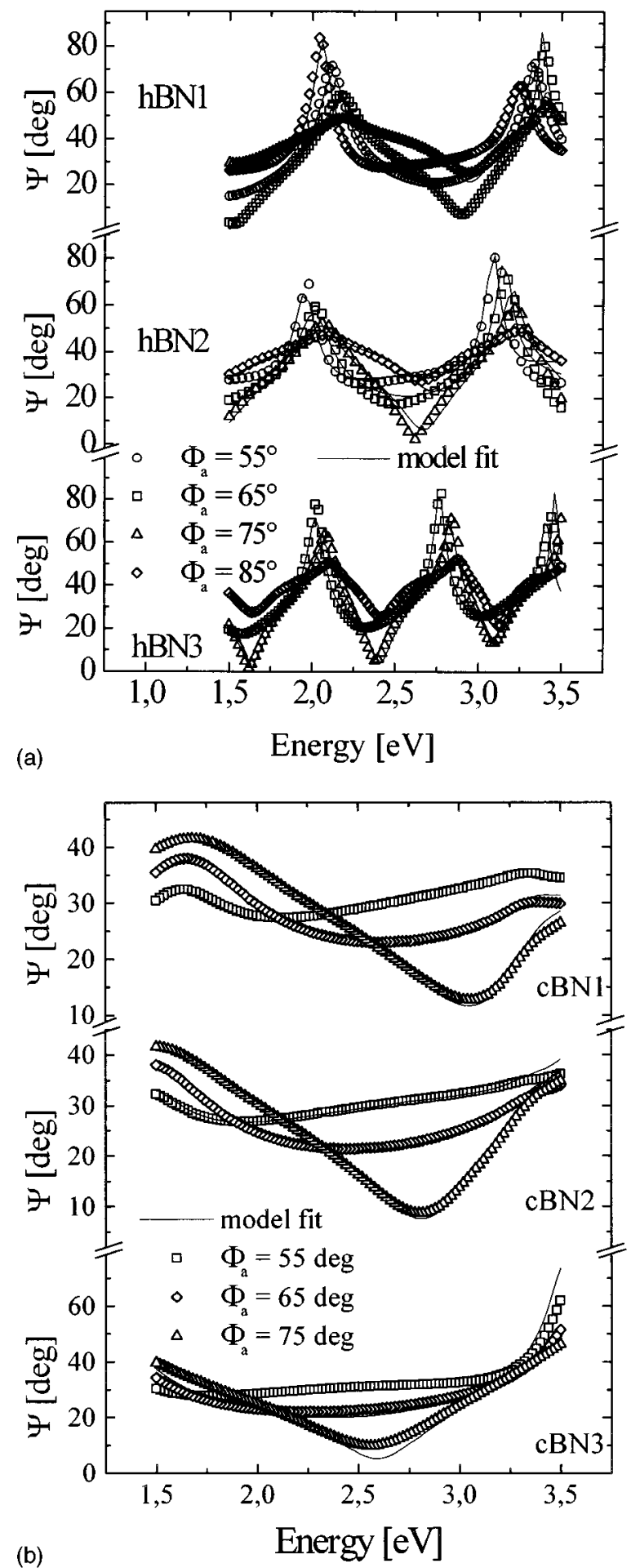

FIG. 3. (a) Experimental (symbols) and generated visible-light gVASE data (solid lines) obtained from the $h$-BN samples ( $\Psi$ only; $\Phi_{a}=55^{\circ}-85^{\circ}$; $h$-BN1: upper panel; $h$-BN2: middle panel; $h$-BN3: lower panel). The generated data were taken from the best multiple sample fit for $n_{e}, n_{o}$, the respective layer thickness and cone angle $\Theta$. (b) Same as (a) for the highcontent $c$-BN thin-film samples $\left(\Phi_{a}=55^{\circ}-75^{\circ}\right) ; c$-BN1: upper panel; $c$-BN2: middle panel; $c$-BN3: lower panel). The generated data were taken from the best sample fit for $n_{c}, f_{c}$, and the respective layer thickness.

microstructure affected infrared optical properties of BN thin films and their spectroscopic ellipsometry characterization will be given elsewhere. ${ }^{36,38}$

The large broadening of the $\omega_{\mathrm{TO} \perp}$ peak in $h$ - $\mathrm{BN} 2$ can be
$\mathrm{BN}$ refractive indices

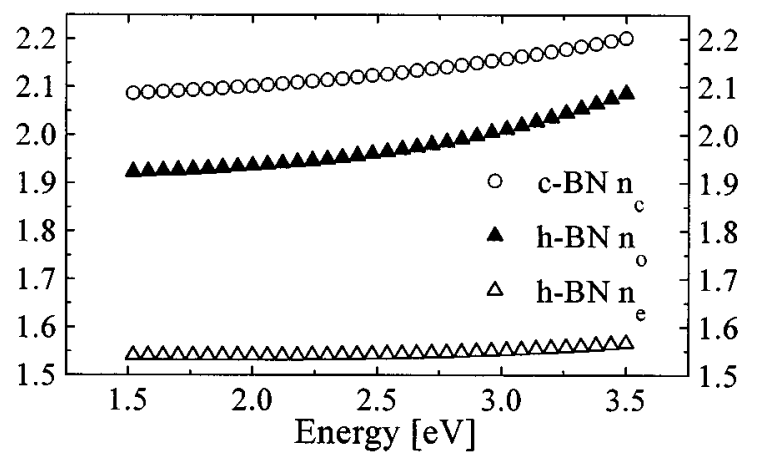

FIG. 4. BN refractive indices obtained from multiple-sample regression analysis fit and visible-light gVASE data $\left(n_{c} c\right.$-BN: open circles, $n_{e} h$-BN: open up-triangles, $n_{o} h$-BN: solid up-triangles).

explained as due to very small grain sizes, as proven by HRTEM and SAD measurements. A strong LO phonon mode resonance is excited at $\omega=\omega_{\mathrm{LO} \|}$ in sample $h$-BN1 and only a small signal due to the TO mode $\left(\omega_{\mathrm{TO} \|}\right)$ can be observed. Sample $h$-BN2 behaves optically isotropic due to randomly oriented grain $c$ axes $\left(\theta=54.7^{\circ}\right)$, and the out-ofplane lattice absorptions virtually disappear. This is inherent to the microstructure-dependent model for the thin-film DF. The difference between the experimental and generated data in $h$-BN3 is probably due to the high water content within the film. The lattice resonance parameters obtained from the best fit are similar for all samples $\left(\omega_{\mathrm{TO} \|} \sim 775 \mathrm{~cm}^{-1}, \omega_{\mathrm{LO} \|}\right.$ $\left.\sim 820 \mathrm{~cm}^{-1}, \omega_{\mathrm{TO} \perp} \sim 1400 \mathrm{~cm}^{-1}, \omega_{\mathrm{LO} \perp} \sim 1585 \mathrm{~cm}^{-1}\right)$ and agree with bulk data.

Figure 5(b) shows generated and experimental data $(\Psi$ only) from the high $c$-BN content samples. A two-layer model was used to account for the $h$-BN nucleation layer. The generated data were obtained from the best fit for the $c$-BN DF parameters $\left(\varepsilon_{0 c}, \omega_{\mathrm{TO} c}, \omega_{\mathrm{LO} c}, \Gamma_{c}\right)$, the $h$-BN parameters as described above, the layer thicknesses $d_{1}$ and $d_{2}$, the $c$-BN volume fraction $f_{c}$, and the nucleation layer cone angle $\Theta$. The double LO mode resonance in $\Psi$ at $\omega \cong 1600 \mathrm{~cm}^{-1}$ is due to the difference in grain $c$-axis orientations between the nucleation layer and the randomly averaged (isotropic) $h$-BN material at the grain boundaries, i.e., two spectrally separated $h$-BN LO phonons excited within the nucleation layer and the high $c$-BN content layer, respectively. ${ }^{36,38}$ The $c$-BN and $h$-BN TO modes are shown by insets. The $c$-BN lattice resonance frequencies $\omega_{\mathrm{TO}}$ $\sim 1070 \mathrm{~cm}^{-1}, \omega_{\mathrm{LO} c} \sim 1300 \mathrm{~cm}^{-1}$ obtained from the best fit are in good agreement with bulk data.

The microstructure-dependent $h$-BN and $c$-BN thin-film fit parameters are summarized in Table I compared to the results obtained from the gVASE data analysis. In contrast to the lack of sensitivity to the nucleation layer optical properties provided by the visible-light gVASE data, the IRSE data contain enough information for their nondestructive characterization.

We emphasize the consistency between the results obtained from gVASE and IRSE data. The single geometrical model for the thin-film dielectric function applied for data analysis for both the infrared and visible spectral range cov- 

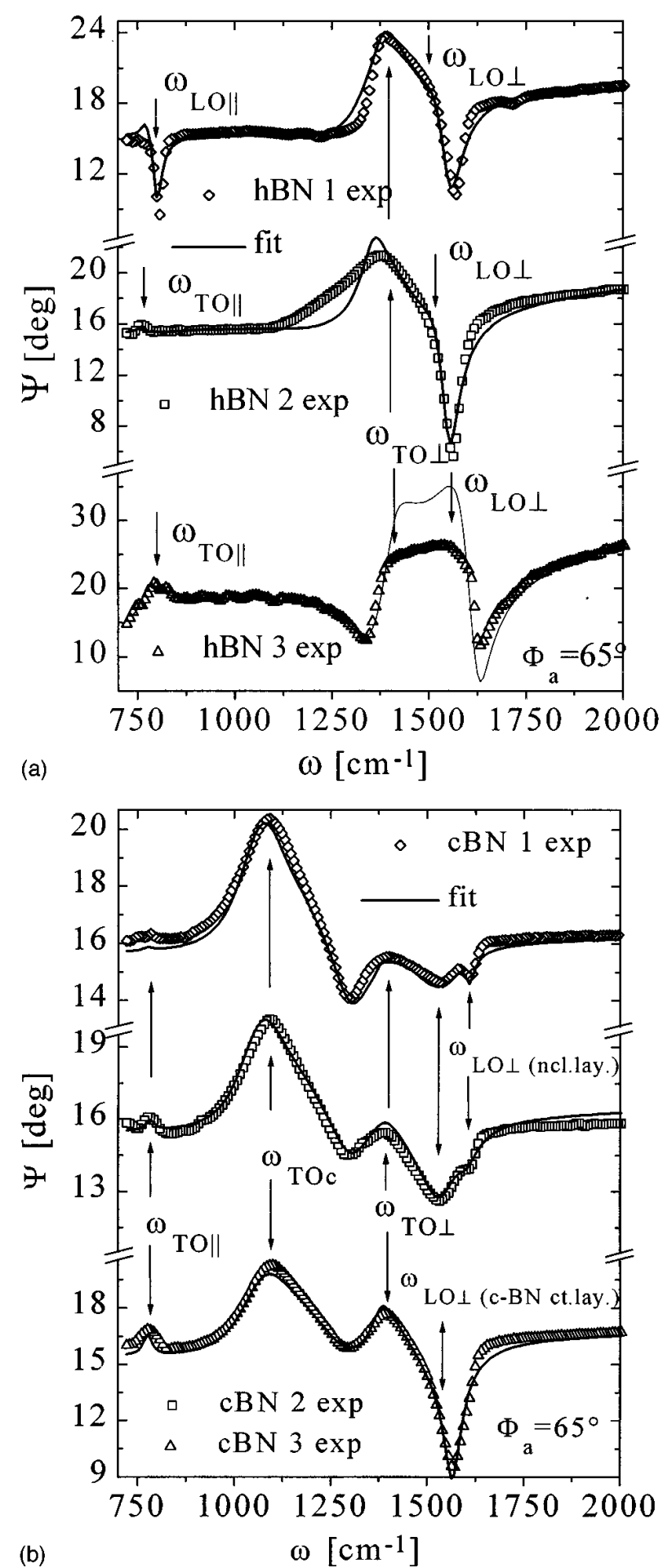

FIG. 5. (a) Experimental (symbols) and generated data (solid lines) obtained from our $h$-BN thin-film samples $\left(\Psi\right.$ only; $\Phi_{a}=65^{\circ} ; h$-BN1: open diamonds; $h$-BN2: open squares, $h$-BN3: open triangles). The generated data were taken from the best fit for $\omega_{\mathrm{TO} \|}, \omega_{\mathrm{LO} \|}, \varepsilon_{0 \|}, \Gamma_{\|}, \omega_{\mathrm{TO} \perp}, \omega_{\mathrm{LO} \perp}, \varepsilon_{0 \perp}$, $\Gamma_{\perp}$, the average grain $c$-axes orientation $\Theta$, and the particular layer thickness. The various $\Psi$ spectra are due to the different grain $c$-axes orientations. (b) Same as (a) for our high $c$-BN content thin-film samples ( $\Phi_{a}=65^{\circ} ; c$-BN1: open diamonds; $c$-BN2: open squares, $c$-BN3: open triangles). The generated data were taken from the best fit for $\omega_{\mathrm{TO} c}, \omega_{\mathrm{LO} c}$, $\varepsilon_{0 c}, \Gamma_{c}, f_{c}$, and the particular layer thickness. The various spectra are due mainly to the different $c$-BN contents $f_{c}$ within the thin films. The double LO mode resonance in $\Psi$ at $\omega \cong 1600 \mathrm{~cm}^{-1}$ is due to the difference inc-axis grain orientations between the nucleation layer and the randomly averaged (isotropic) $h$-BN material at the grain boundaries, i.e., two spectrally separated $h$-BN LO phonons excited within the nucleation layer and the high $c$-BN content layer, respectively. ers mechanical as well as electronical properties of the polycrystalline thin films. The model predicts characteristic BN thin-film optical anisotropy in the visible spectral range. In turn, the same microstructure then causes a distinct infrared reflectivity behavior due to microstructure dependent longitudinal optical thin-film phonon modes. These modes are observable only in thin films with thickness less than a fraction of the incident-light wavelength. This is known as the Berreman effect. The volume fractions of $c$-BN within the high $c$-BN content thin films $f_{c}$ obtained from gVASE and IRSE data analysis using the Bruggeman effective medium approximation differ slightly. We believe that the gVASE data overestimate the $c$-BN content by about $5 \%$. The thickness and average $c$-axis grain orientation obtained from our ellipsometric model are consistent with HRTEM and SAD investigations, performed on the same samples.

\section{CONCLUSIONS}

We have presented a microstructure-dependent model for the thin-film visible and infrared anisotropic effective dielectric function to explain ellipsometric data acquired from various samples. We find that IRSE is very sensitive to phase and microstructural properties of $\mathrm{BN}$ thin films. Generalized VASE investigations have demonstrated that the $h$-BN visible-light thin-film effective refractive index is anisotropic, and changes from uniaxially positive to negative due to growth parameter dependent average $c$-axis grain orientations within the pure-phase $h$-BN samples. We obtain the average orientation of the $h$-BN grain $c$ axes in pure-phase $h$-BN layers, as well as the amount of $c$-BN within high $c$-BN content layers. A small noncubic nucleation layer causes splitting of the thin-film LO resonance frequencies in the in-plane resonance region. The microstructure-relevant parameters obtained from best sample analysis fits for the IRSE and gVASE data are identical, and agree with those obtained from HRTEM and SAD investigations.

\section{ACKNOWLEDGMENTS}

The authors wish to acknowledge G. Wagner at University Leipzig and M. Röder at Technical University Chemnitz-Zwickau for TEM investigations. They thank B. Rheinländer at University Leipzig and Th. Welzel at Technical University Chemnitz-Zwickau for helpful support. They offer gratitude to Mick DeVries for his assistance during the experiments, and thank the Center for Microelectronic and Optical Materials Research of the University of Nebraska-Lincoln for financial support during part of the work.

${ }^{1}$ N. Tanabe, T. Hayashi, and M. Iwaki, Diam. Relat. Mater. 1, 883 (1992).

${ }^{2}$ K. F. McCarty, P. B. Mirkarimi, D. L. Medlin, T. A. Friedmann, and J. C. Barbour, Diam. Relat. Mater. 5, 1519 (1996).

${ }^{3}$ H. Hofsäss, C. Ronning, U. Griesmeier, S. Reinke, and M. Kuhr, Appl. Phys. Lett. 67, 46 (1995).

${ }^{4}$ A. Schütze, K. Bewilogua, H. Lüthje, and S. Kouptsidis, Diam. Relat. Mater. 5, 1130 (1996)

${ }^{5}$ S. Reinke, M. Kuhr, W. Kulisch, and R. Kassing, Diam. Relat. Mater. 4, 272 (1995).

${ }^{6}$ A. K. Ballal, L. Salamanca-Riba, C. A. Taylor III, and G. L. Doll, Thin Solid Films 224, 46 (1993). 
${ }^{7}$ K.-L. Barth, A. Neuffer, J. Ulmer, and A. Lunk, Diam. Relat. Mater. 5, 1270 (1996).

${ }^{8}$ D. J. Kester, K. S. Ailey, D. J. Lichtenwalner, and R. F. Davis, J. Vac. Sci. Technol. A 12, 3074 (1994).

${ }^{9}$ D. L. Medlin, T. A. Friedmann, P. B. Mirkarimi, P. Rez, M. J. Mills, and K. F. McCarty, J. Appl. Phys. 76, 295 (1994).

${ }^{10}$ M. Schubert, B. Rheinländer, E. Franke, H. Neumann, J. Hahn, M. Röder, and F. Richter, Appl. Phys. Lett. 70, 1819 (1997).

${ }^{11}$ W. L. Zhou, Y. Ikuhara, M. Murakawa, S. Watanabe, and T. Suzuki, Appl. Phys. Lett. 66, 2490 (1995).

${ }^{12}$ A. S. Rozenberg, Yu. A. Sinenko, and N. V. Chukanov, J. Mater. Sci. 28, 5675 (1993).

${ }^{13}$ T. Kuzuba, K. Era, T. Ishii, and T. Sato, Solid State Commun. 25, 863 (1978).

${ }^{14}$ D. M. Hoffman, G. L. Doll, and P. C. Eklund, Phys. Rev. B 30, 6051 (1984).

${ }^{15}$ M. F. Plass, W. Fukarek, S. Mändl, and W. Möller, Appl. Phys. Lett. 69, 46 (1996).

${ }^{16}$ R. Geick, C. H. Perry, and G. Rupprecht, Phys. Rev. 146, 543 (1966).

${ }^{17}$ P. J. Gielisse, S. S. Mitra, J. N. Plendl, R. D. Griffis, L. C. Mannsur, R. Marshall, and E. A. Pascoe, Phys. Rev. 155, 1039 (1967).

${ }^{18}$ M. I. Eremets, M. Gauthier, A. Polian, J. C. Chervin, J. M. Besson, G. A. Dubitskii, and Ye. Ye. Semenova, Phys. Rev. B 52, 8854 (1995).

${ }^{19}$ M. Okamoato, Y. Utsumi, and Y. Osaka, Jpn. J. Appl. Phys., Part 1 31, 3455 (1992).

${ }^{20}$ R. Poerschke and O. Madelung, Semiconductors Group IV Elements and III-V Compounds (Springer, Berlin, 1991).

${ }^{21}$ S. L. Ren, A. M. Rao, P. C. Eklund, and G. L. Doll, Appl. Phys. Lett. 62, 1760 (1993).
${ }^{22}$ D. R. McKenzie, W. D. McFall, H. Smith, B. Higgins, R. W. Boswell, A. Durandet, B. W. James, and J. S. Falconer, Nucl. Instrum. Methods Phys. Res. B 106, 90 (1995).

${ }^{23}$ E. Pascual, J. L. Andújar, S. Gimeno, A. Lousa, A. Bosch, M. El Kasmi, and E. Bertran, Diam. Relat. Mater. 5, 539 (1996).

${ }^{24}$ C. Quirós, P. Prieto, J. F. Trigo, E. Elizalde, and J. M. Sanz, Nucl. Instrum. Methods Phys. Res. B 112, 275 (1996).

${ }^{25}$ T. H. Yuzuriha and D. W. Hess, Thin Solid Films 140, 199 (1986).

${ }^{26}$ W. Baronian, Mater. Res. Bull. 7, 119 (1972).

${ }^{27}$ M. Murakawa, S. Watanabe, and S. Miyake, Thin Solid Films 226, 82 (1993).

${ }^{28}$ D. C. Cameron, M. Z. Karim, and M. S. J. Hashmi, Thin Solid Films 236, 96 (1993).

${ }^{29}$ A. Zunger, A. Katzir, and A. Halperin, Phys. Rev. B 13, 5560 (1976).

${ }^{30}$ N. E. Christensen and I. Gorczyca, Phys. Rev. B 50, 4397 (1994).

${ }^{31}$ R. M. A. Azzam and N. M. Bashara, Ellipsometry and Polarized Light (North-Holland, Amsterdam, 1987).

${ }^{32}$ M. Schubert, B. Rheinländer, B. Johs, C. M. Herzinger, and J. A. Woollam, J. Opt. Soc. Am. A 13, 875 (1996).

${ }^{33}$ J. Hahn, M. Friedrich, R. Pintaske, M. Schaller, N. Kahl, D. R. T. Zahn, and F. Richter, Diam. Relat. Mater. 5, 1103 (1996).

${ }^{34}$ D. E. Aspnes and J. B. Theeten, Phys. Rev. B 20, 3292 (1979).

${ }^{35}$ M. Schubert, Phys. Rev. B 53, 4265 (1996).

${ }^{36}$ E. Franke, H. Neumann, M. Schubert, T. E. Tiwald, J. A. Woollam, and J. Hahn, Appl. Phys. Lett. 70, 1668 (1997).

${ }^{37}$ D. W. Berreman, Phys. Rev. 130, 2193 (1963).

${ }^{38}$ M. Schubert, B. Rheinländer, E. Franke, H. Neumann, T. E. Tiwald, J. A. Woollam, J. Hahn, and F. Richter (unpublished). 